\title{
MESOSCALE ACOUSTICAL CYLINDRICAL SUPERLENS
}

\author{
Igor Minin, ${ }^{a}$, Oleg Minin \\ National Research Tomsk State University, 634050, Tomsk, Russia
}

\begin{abstract}
We demonstrate experimentally for the first time the acoustojet (acoustic jets) formed from acoustic plane wave scattering by a penetrable cylindrical particle with dimensions of several wavelengths. It acts as a superlens with subwavelength localization of acoustical wave. During the scattering by elastic solid particles, additional internal shear waves are excited due to modes conversion. This mechanism allows achieving sharp focusing in the near-field zone. Such mesoscale single particle cylindrical lens may be considered as acoustic metamaterials free superlenses with resolution beyond the diffraction limit.
\end{abstract}

\section{Introduction}

Acoustic lenses find applications in various areas ranging from nondestructive detection to biomedical imaging and even noninvasive cancer treatment. One of the leading trends in the development of modern devices is miniaturization and integration. It is therefore of paramount significance to realize an acoustic lens that has a compact (mesoscale) size, a subwavelength resolution and a high transmission efficiency and thus should have deep implication for the acoustic field. However, its realization remains challenging due to the tradeoff between high refractive-index and impedance-mismatch.

The first acoustical lens has been probably described in $[1,2]$. Last century several acoustic lens designs were considered: planar acoustic lens structures based on Fresnel diffraction [3], sonic-crystal slab composed of a two-dimensional square-array of aluminum rods in air [4], 3D phononic crystal [5, 6], metamaterial based acoustic gradient index (GRIN) lenses [7, 8], hyperbolic GRIN lens [9, 10], etc. It could be noted that GRIN lens are far less prevalent in practice due to the difficulty in finding real materials with desired parameters, since natural materials are generally unable to provide slow sound velocity and high mass density simultaneous In 1991 for the Soret geometry of zone plate shock diffraction and focusing has been simulated by referencing a characteristic wavelength of an attenuated shock pulse [11]. Strong focusing of a plane ultrasound wave by a plano-concave lens was investigated in [12]. Many of modern approaches use Helmholtz resonators or metamaterial based split-ring type resonators [13]. However, these metamaterials are all bulk materials and much larger than the working wavelength, which is to the disadvantage of the miniaturization and integration of acoustic devices. Brief review of acoustical artificial lenses was given in Ref. [14]. However, existing methods of subwavelength focusing in

\footnotetext{
a Corresponding author: prof.minin@gmail.com
} 
acoustics are usually based on new artificial materials, or require complex signal processing methods.

It is well known, that due to diffraction theory, the resolution of ultrasound imaging is limited at best to a half-wavelength and features below this value cannot be resolved. Shorter wavelength can be used, but to the cost of a higher attenuation and lower penetration depth, which rapidly reduces the scope of the imaging technique. To date, this "resolution versus penetration" paradigm annihilates the quest for ultrasound imaging deep into target.

In the optical frequency range, optical jet-like structures for small dielectric particles were investigated at least since 1987 [15]. The well-known by now so-called photonic nanojets (PNJ) allow to form a localized hot-spot in subwavelength area in light scattering by low loss dielectric particles [16].

On the other hand, in the linear mode it should be expected that methods of sub-wave focusing, based on photonic jet phenomenon, can be successfully applied to the acoustic range as well. Formally this can be observed on the analogy between the wave equations, specifying acoustic and electromagnetic scattering processes [17]. Thus, PNJ effect have motivated us to propose the concept of acoustic jets, called "acoustojets" [18, 19]. It has been theoretically predicted for the first time $[18,19]$ that the existence of acoustic analogue of PNJ phenomenon is possible, providing an opportunity for subwavelength localization of acoustic field in shadow area of arbitrary 3D penetrable mesoscale particle. It is important to note that there is a principle difference between optical and acoustical materials such as existence of a shear speed of sound, i.e. acoustic materials are anisotropic due to the two speeds of sound [20,21]. Recently, the phenomenon of acoustojet formation was experimentally verified for spherical Rexolite particle, immersed in water [22].

\section{Cylindrical acoustojet}

In this report, for the first time we experimentally demonstrate that highly intense acoustic beam with subwavelength waist is produced in the scattering by a mesoscale penetrable cylindrical particle. It is well known that metallic spherical particles have low acoustic absorption. In the case of the rigid particle pressure waves do not penetrate inside it, while in the case of the penetrable particle both cubical and spherical shape pressure waves may travel through it and can excite internal modes [23]. From our previous simulation results [18-20] it follows that metallic spherical particles (made of lead, stainless steel, brass, etc.) can produce acoustojets, but due to an acoustic impedance mismatch, the intensity is relatively low. Due to this reason for further experimental investigations we selected polymer mesoscale particles, like ABS plastic (black), which has perfect acoustic impedance matching with water (see the Table 1 [24]).

Table 1. Parameters of the used materials.

\begin{tabular}{|l|l|l|l|}
\hline Material & Sound velocity, $\mathrm{m} / \mathrm{s}$ & Density, $\mathrm{g} / \mathrm{sm}^{3}$ & $\begin{array}{l}\text { Acoustic } \\
\text { impedance }\end{array}$ \\
\hline Water & 1490 & 0.998 & 1.487 \\
\hline Rexolite & 2337 & 1.04 & 2.430 \\
\hline Plexiglas & 2670 & 1.18 & 3.150 \\
\hline ABS Plastic (black) & 2250 & 1.05 & 2.362 \\
\hline
\end{tabular}




\section{Experimental equipment}

Let's briefly consider an experimental setup for cylindrical acoustojets measurements. The basic equipment was similar to that used in Ref. [25] - see Figure 1. A signal generator GZ112 was used to excitation of transducer. The transducer assembly with the nominal thickness-resonance of ceramic emitter $1.092 \mathrm{MHz}$ was fixed centrally into one of the square faces of a rectangular glass-walled tank, dimensions $20 \times 20 \times 30 \mathrm{~cm}$, with the transducer axis horizontal and parallel to the length of the tank.

To measure the acoustic parameters in the focusing area we used a calibrated [26, 27] broad band needle-like hydrophone GUZ-2 [28]. A 3D printed black ABS cylinder with diameter of 4 wavelength was suspended by a thin $(0.08 \mathrm{~mm})$ fishing line and placed along the central part of transducer. The position of the hydrophone was determined by a threecoordinate system of positioning, controlled by a computer, similar to Ref. [29]. Such measurements were carried out with a spatial step of $0.5 \mathrm{~mm}$ in an area of $60 \times 60 \mathrm{~mm}$. The transverse and longitudinal distributions of the acoustic pressure were measured.

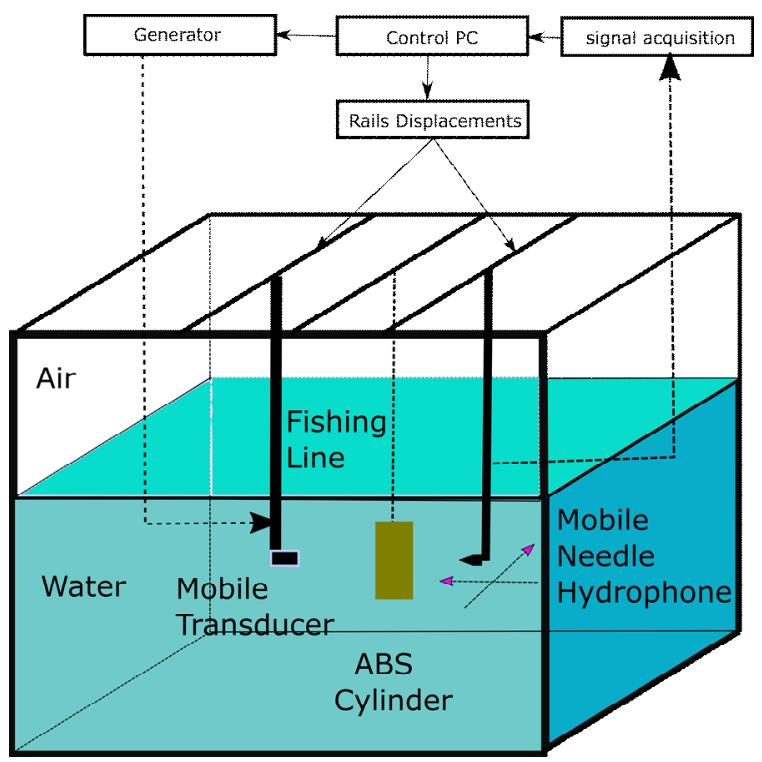

Fig. 1. Schematic of the experimental setup.

\section{Simulations}

A simulation model which mimics an experimental physical phenomenon could be a primary model to start different designs for improvement. We first simulated the cylindrical acoustic particle-lens using Finite Element Method (FEM) software Comsol Multiphysics. Boundary conditions near the surface of glass-walled tank were set as water acoustic impedance in order to avoid reflections of the propagation wave. We used a free tetrahedral mesh with maximum element size $\lambda / 5$ for the free-space regions, and $\lambda / 5 / 1.5$ for the particle. Additionally, we used a free triangular mesh with maximum element size $\lambda / 20$ for the z-plane to obtain Figures of better resolution - the need for very fine meshes in acoustic jet numerical simulations is a known problem in optics, and an even finer mesh is required for obtaining the field's spatial derivatives to obtain the forces. The properties of the material were set through the longitudinal and shear velocities of sound. 
Modeling the interaction of ultrasound with such a structure is a complex problem, and further modeling the resultant wave behavior after the interaction is also challenging. The complexity of these problems arise from parameters such as the number and type of boundaries involved, the number of reflections accounted for, the number and type of wave modes interacting, and how the focal spot is defined [30].

\section{Experimental results}

Similar to optics $[16,31]$, the analysis of acoustojet is characterized by such main parameters of ultrasound beams as peak intensity, its position along $\mathrm{Z}$ axis, full width at half maximum (FWHM), full length at half maximum (FLHM) at $-3 \mathrm{~dB}[22,32]$. The experimental measurement of FWHM and FLHM are shown in the Figure 2. The pressure distribution along the wave propagation direction was measured from a distance of $0.5 \lambda$ from the shadow surface of the cylinder to eliminate the influence of the probe. The enhancement of the measured acoustojet is 1.8 with sidelobes under 1.2. The similar results (with lower gain) were observed for cylindrical particles illuminated from a flat surface.

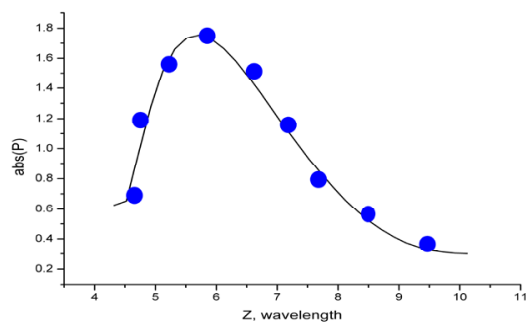

a)

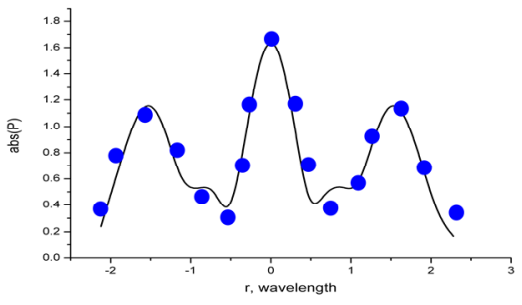

b)

Fig.2. The experimental result for the acoustojet relative pressure intensity in the xz (a) and xy (b) plane. The solid lines present the results of numerical simulations in Comsol Multiphysics software. The derived length of acoustojet FLHM is about $2.5 \lambda$ and FWHM is less than $0.62 \lambda$.

\section{Conclusion}

In conclusion, we have demonstrated for the first time that acoustojets (acoustic jets) formed by acoustic plane wave scattered by penetrable cylindrical particle lens with the diameter of four wavelengths may be used for subwavelength localization of acoustical waves. This work presents a quantitative comparison between experimental and simulated acoustic fields of acoustical cylindrical particle lens. In the acoustic wave scattering by elastic solid mesoscale particles shear waves are excited due to mode conversion leading to a stronger localization effect and this is a fundamental difference from optical nanojet phenomenon.

Such mesoscale particles can be considered as an acoustic superlenses with resolution beyond the diffraction limit and, thus, can be used in acoustical tweezers for particle manipulation, medical diagnostics, non-destructive testing, sensors, acoustical microscopy etc, due to subwavelength resolution and high energy gradient.

Our results can be further extended to the particles made of different materials and of various shapes with the attention to the feasibility of acoustical subwavelength focusing with a single particle lens (both spherical and cuboid) from artificial periodic structures, core-shell particle with a liquid core (including blood) and a coating layer, metamaterials, particles with pupil mask and the whole other arsenal of methods previously developed in the optical range [26]. 


\section{Acknowledgments}

I.V.M. acknowledges the support of the Russian Scientific Foundation, grant 17-79-20051 (test object design and characterization), O.V.M acknowledges the support of the Mendeleev scientific fund of Tomsk State University (experimental setup). O.V.Minin and I.V.Minin contributed equally to this work.

\section{References}

[1] W.E.Kock, F.K. Harvey, Jour. Acous. Soc. Amer. 21, 471 (1949)

[2] G.L. Augspurger, Electronics World 52 (1962)

[3] B. Hadimioglu, E.G. Rawson, R. Lujan, M. Lim, J.C. Zesch, B.T. Khuri Yakub, F.C., Quate Proc. IEEE Ultrasonics Symp. 1, 579 (1993)

[4] T. Miyashita, R.Taniguchi, H. Sakamoto, Proc. WCU, Paris, Sep. 7-10, 911 (2003)

[5] J.T. Welter, S. Sathish, D.E. Christensen, P.G. Brodrick, J.B. Heebl, M.R. Cherry, J. Acoust. Soc. Am. 130, 5 (2010)

[6] S. Alagoz, Kaya O. Adem, B.B. Alagoz, Applied Acoustics. 70, 1400 (2009)

[7] V. Romero-Garcia, A. Cebrecos, R. Pico, V.J. Sanchez-Morcillo, L.M. Garcia-Raffi, a J.V. Sanchez-Perez, Applied Physics Letters. 103, 264106 (2013)

[8] Li Y., Yu G., Liang B., Zou X., Li G., Su Cheng and Cheng J. Sci. Rep. 4, 6830 (2014)

[9] W. Wang, Y. Xie, A. Konneker, B. Popa, S.A. Cummer. Applied Physics Letters. 105, 101904 (2014)

[10] M. Moleron, M. Serra-Garcia, C. Daraio, Applied Physics Letters 105, 114109 (2014)

[11] I.V. Minin, O.V. Minin, V.F. Minin, Book of abstracts the 18th Int. Symp.on Shock waves, Ed by K.Takayama (1991)

[12] M. Akiyama, T. Kamakura. Acoust. Sci. \& Tech. 26, 3 (2005)

[13] W.M. Robertson, US Patent 2016/0111080, A1 (2016)

[14] I.V. Minin, O.V.Minin, Proc. of APEIE, 2016 13th Int. Sci.-Tech. Conf. 136 (2016)

[15] D.S. Benincasa, P.W. Barber, J.Z. Zhang, W.F. Hsieh, R.K. Chang, Applied Optics 26, 1348 (1987)

[16] B.S. Lukiyanchuk, R. Paniagua-Domínguez, I.V. Minin, O.V. Minin, W. Zengbo. Optical material express 7, 1820 (2017)

[17] L. Nicolas, M. Furstoss, M.A. Galland. The European Physical Journal Applied Physics 4, 95 (1998)

[18] I.V. Minin, O.V. Minin, arXiv, 1604.08146 (2016)

[19] O.V. Minin, I.V. Minin, Optical and Quantum Electronics 49, 54 (2017)

[20] J.H. Lopes, J.P. Leao-Neto, I.V. Minin, O.V. Minin, G.T. Silva, Proc. 2nd ICA2016, 1 (2016)

[21] B.D. Tartakovski, Akusticheskij Zhurnal 7, 349 (1961)

[22] J.H. Lopes, M.A.B. Andrade, J.P. Leao-Neto, J.C. Adamowski, I.V. Minin, G.T. Silva Physical Review Applied 8, 024013 (2017)

[23] A. Maurel, J.-F. Mercier and Felix, S. J. Acoust. Soc. Am. 135, 165 (2014)

[24] Selfridge A.R. IEEE Transactions on Sonics and Ultrasonics, SU-32, 381 (1985)

[25] Neppiras E.A. and Coakley W.T. Journal of Sound and Vibration 45, 341 (1976)

[26] Papadakis P., Taroudakis M., Sturm F., Sanchez P., and Sessarego J-P. Acta Acustica united with Acustica 94, 676 (2008)

[27] IEC 60565:2006, Underwater acoustics - Hydrophones - Calibration in the frequency range 0,01 Hz to $1 \mathrm{MHz}$ (International Electrotechnical Commission, International Standard, 2006) 
[28] A. M. Enyakov Development of methods and tools for metrological support of hydroacoustic measurements in the frequency range from 0.5 to $15 \mathrm{MHz}$ (Degree of the Doctor of Technical Sciences Thesis (in Russian), Mendeleevo, 2007)

[29] A. Cebrecos, V. Romero-García, R. Picó, V.J. Sánchez-Morcillo, M. Botey, R. Herrero, Y.C. Cheng and K. Staliunas, J Phys D Appl Phys 48, 025501 (2014)

[30] E. Twerdoski, M. Von Buttlar, A. Habib, R. Wannemacher, W. Grill, ECNDT 2006 - Fr.1.8.4 URL: http://www.ndt.net/article/ecndt2006/ doc/Fr.1.8.4.pdf

[31] I.V. Minin and O.V. Minin, Diffractive Optics and Nanophotonics: Resolution Below the Diffraction Limit ( Springer, London, 2016)

[32] S. Yang, W. Qin, H. Guo, T. Jin, N. Huang, M. He, and L. Xi, Biomedical optics express 8, 2756 (2017) 